\title{
Novel Candidate Biomarkers of Chemoradiosensitivity in Esophageal Squamous Cell Carcinoma: A Systematic Review
}

\author{
Yusuke Sato $^{a}$ Satoru Motoyama ${ }^{a}$ b Hajime Saito ${ }^{a}$ Yoshihiro Minamiya ${ }^{a}$ \\ Departments of a Thoracic Surgery, and ${ }^{b}$ Comprehensive Cancer Control, Akita University Graduate \\ School of Medicine, Akita, Japan
}

\section{Key Words}

Esophageal cancer · Squamous cell carcinoma · Chemoradiosensitivity · Biomarker . Prognosis

\begin{abstract}
There is no doubt that, along with surgery, chemoradiotherapy is an important treatment for esophageal squamous cell carcinoma (ESCC). Patients who respond well to chemoradiotherapy obtain great benefits toward overcoming their cancer, and so a more favorable prognosis. On the other hand, patients who do not respond well have wasted valuable time and experienced severe toxicity and seriously diminished quality of life, only to have their cancer recur with an unfavorable prognosis. For this reason, a reliable biomarker of chemoradiosensitivity in ESCC has long been sought. In this review, we will enumerate recently reported candidate biomarkers of chemoradiosensitivity in ESCC that have the potential for future clinical application.

(C) 2016 S. Karger AG, Basel
\end{abstract}

\section{Introduction}

According to the WHO, in 2014 esophageal cancer was the 6th most common cancer globally [1]. In fact, thoracic esophageal squamous cell carcinoma (ESCC) is the most common form of cancer in many regions of the world, and is characterized by an extremely poor prognosis and rapid clinical progression $[2,3]$. Nonetheless, thanks to recent advances in surgical technique, postoperative management and chemoradiotherapy (CRT), the outcomes of these patients have improved [4-7]. Still, the 5-year survival rate among ESCC patients remains low, and further developments in this field are needed.

There is no doubt that, along with surgery, CRT is an important treatment strategy for advanced ESCC [2-7]. Patients who respond well to CRT obtain great benefits toward over- 
Sato et al.: Novel Candidate Biomarkers of Chemoradiosensitivity in Esophageal

Squamous Cell Carcinoma: A Systematic Review

coming their cancer, and so obtain a more favorable prognosis. On the other hand, patients who do not respond well (e.g., when the tumor does not show chemoradiosensitivity) have wasted valuable time and experienced severe toxicity and seriously diminished quality of life, only to have their cancer recur with an unfavorable prognosis [4-8]. For this reason, a reliable marker of chemoradiosensitivity in ESCC has long been sought [8].

In an excellent review, Okumura et al. [9] discussed biomarkers identified between 1980 and 2011 that are potentially predictive of the ESCC response to neoadjuvant CRT. Those authors concluded that p53 is the most important candidate biomarker. However, no biomarker, including p53, has yet been widely accepted for clinical application, nor has one been mentioned as useful for predicting chemoradiosensitivity in the Guidelines for Diagnosis and Treatment of Carcinoma of the Esophagus in Japan [10]. This is because there is still not sufficient evidence of the usefulness of these biomarkers for that purpose.

The purpose of this systematic review article is to enumerate candidate biomarkers reported between 2011 and early 2015 as predictive of chemoradiosensitivity and discuss their potential for future clinical application in ESCC patients.

\section{Methods}

Literature Search

A systematic literature search was performed using PubMed and Google Scholar to evaluate the predictive value of novel candidate biomarkers of chemoradiosensitivity in patients with ESCC. The search strategy was to use combinations of the search terms 'esophageal cancer', 'squamous cell carcinoma', 'chemoradiotherapy', 'chemoradiosensitivity', 'prognosis' and 'biomarker' to identify papers published between 2011 and August 25, 2015. The search was performed repeatedly until no new relevant articles were found. The reference lists of the identified studies were then checked for additional relevant studies, including in vitro assays.

\section{Inclusion/Exclusion Criteria}

The studies included met the following criteria: (1) patients were diagnosed with ESCC based on pathological examination; (2) the biomarker was examined before treatment; (3) patients were treated with CRT before, after or in the absence of surgery; (4) the relationship between biomarker expression and any prognosis [overall survival (OS), disease-specific survival (DSS) and disease-free survival (DFS)] was evaluated, and (5) full-length studies in English. Exclusion criteria were as follows: (1) reviews, letters, case reports; (2) patients were diagnosed with adenocarcinoma or head and neck cancer based pathological examination, and (3) the biomarker was examined during or after treatment. Overlapping articles were excluded by examining the authors' names and affiliations in each publication.

\section{Literature Searches and Study Characteristics}

The search strategy generated 250 references, including 45 in PubMed and 205 in Google Scholar. Of those, only 32 potentially eligible studies were identified. We excluded no studies due to failure to report available data. We thus included 32 full-length articles that met the inclusion criteria. The selection process used for the articles is illustrated in the PRISMA 2009 flow diagram shown in figure 1. We classified the biomarkers based on the testing approach used by the authors, 'blood examination' or 'tissue examination'. We further classified the biomarkers such as 'positive biomarker', 'negative biomarker' and 'combination of biomarkers' in both testing approaches. The classified candidate biomarkers are summarized in tables 1 and 2.

\section{Positive Biomarkers in Blood Examination}

Insulin-like growth factor binding protein-3 (IGFBP-3) is a key regulatory molecule in the IGF axis and functions in a tissue-specific way as both a tumor suppressor and promoter [11]. Zhao et al. [12] reported that ESCC patients carrying the IGFBP-3 rs2854744 GG or GC 
Sato et al.: Novel Candidate Biomarkers of Chemoradiosensitivity in Esophagea

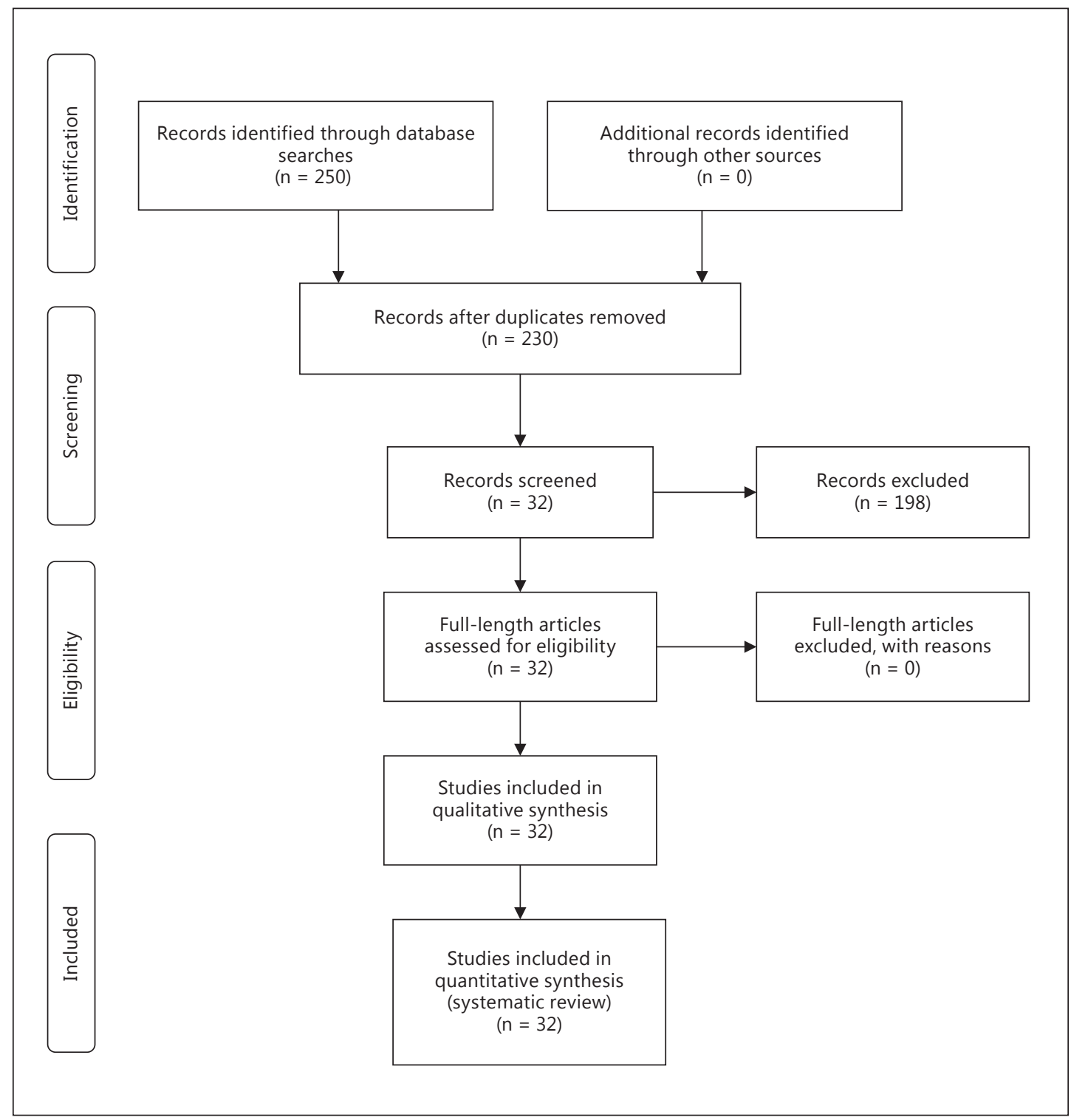

Fig. 1. The selection process used for the articles is illustrated in the PRISMA 2009 flow diagram.

genotype have a significantly better response to CRT and longer OS than those with the CC genotype. On the other hand, we previously reported that IGFBP-3 is highly expressed in ESCC cells deemed highly susceptible to radiotherapy based on cDNA microarray analysis [13]. Moreover, knocking down IGFBP-3 expression in radiosensitive ESCC cells using siRNA significantly reduced radiosensitivity. However, the relationship between the IGFBP-3 SNP and its expression in ESCC remains to be clarified.

Tumor necrosis factor- $\alpha$ (TNF- $\alpha$ ), an important proinflammatory cytokine, is produced by activated macrophages. TNF- $\alpha$ and its superfamily members are well known to have both beneficial and harmful effects. Although TNF- $\alpha$ was discovered as a cytokine able to kill tumor cells, it can also stimulate cell proliferation, invasion and metastasis [14]. Omatsu et al. [15] found that the TNF- $\alpha-857 \mathrm{C}>\mathrm{T}$ genotype is predictive of long-term survival in Japanese ESCC patients treated with definitive CRT. 
Sato et al.: Novel Candidate Biomarkers of Chemoradiosensitivity in Esophageal

Squamous Cell Carcinoma: A Systematic Review

Table 1. Novel candidate biomarkers in blood that are predictive of chemoradiosensitivity in ESCC

\begin{tabular}{|c|c|c|c|c|c|c|}
\hline Biomarker & Major function & $\begin{array}{l}\text { Sample } \\
\text { size }\end{array}$ & Method & Predictive value of the sensitivity & First author [Ref.] & Year \\
\hline $\begin{array}{l}\text { Positive } \\
\text { IGFBP-3 }\end{array}$ & IGF transport & 110 & blood PCR & rs2854744 GG + GC genotype & Zhao [12] & 2015 \\
\hline TNF- $\alpha$ & cell apoptosis & 46 & blood PCR & TNF- $\alpha 857 \mathrm{C}>\mathrm{T}$ & Omatsu [15] & 2013 \\
\hline Hemoglobin & oxygen transport & 123 & blood exam & $\mathrm{Hb}>13 \mathrm{~g} / \mathrm{dl}$ & Hamai [16] & 2014 \\
\hline Albumin & $\begin{array}{l}\text { colloidal osmotic } \\
\text { pressure }\end{array}$ & 74 & blood exam & $\mathrm{Alb}>3.5 \mathrm{~g} / \mathrm{dl}$ & Hamai [17] & 2013 \\
\hline \multicolumn{7}{|l|}{ Negative } \\
\hline VEGF & tumor angiogenesis & $\begin{array}{l}79 \\
49 \\
\end{array}$ & $\begin{array}{l}\text { serum PCR } \\
\text { blood PCR }\end{array}$ & $\begin{array}{l}\text { VEGF-A(-), TGF- } \beta_{1}(-) \\
\text { VEGF } 634 \text { C/G }\end{array}$ & $\begin{array}{l}\text { Cheng [19] } \\
\text { Tamura [20] }\end{array}$ & $\begin{array}{l}2014 \\
2012 \\
\end{array}$ \\
\hline XPA, XPC & DNA repair & 501 & blood PCR & XPA 5'UTR, XPC K939Q wild type & Yang [23] & 2013 \\
\hline IL-6R & cytokine receptor & 64 & serum PCR & IL-6R (-) & Makuuchi [25] & 2013 \\
\hline $\begin{array}{l}\text { Combination } \\
\text { Octanoylcarnitine, } \\
\text { lysoPC(16:1), } \\
\text { decanoylcarnitine }\end{array}$ & metabolomics & 44 & RRLC-MS & $\begin{array}{l}\text { octanoylcarnitine }(-), \\
\text { lysoPC }(16: 1)(-) \text {, decanoyl- } \\
\text { carnitine }(-)\end{array}$ & $\mathrm{Xu}[27]$ & 2013 \\
\hline
\end{tabular}

RRLC-MS = Rapid resolution liquid chromatography-mass spectrometry.

Hemoglobin is one of the most routinely assayed blood components in clinical practice. However, a multivariable analysis performed by Hamai et al. [16] revealed that the pretreatment hemoglobin level is an independent predictor of the response to neoadjuvant CRT, and that patients with hemoglobin levels $<13 \mathrm{~g} / \mathrm{dl}$ had a significantly higher rate of poor responders and worse 5-year OS. Those investigators also reported in a separate study that a pretreatment serum albumin level $>3.5 \mathrm{~g} / \mathrm{dl}$ was independently associated with prolonged survival [17].

\section{Negative Biomarkers in Blood Examination}

Vascular endothelial growth factor (VEGF)-A is a potent signaling molecule that contributes to tumor angiogenesis and hematogenous spread of disease [18]. Cheng et al. [19] demonstrated that among ESCC patients treated with neoadjuvant CRT followed by esophagectomy, those exhibiting high serum levels of VEGF-A and transforming growth factor (TGF)$\beta_{1}$ had a significantly worse pathological response and worse DSS. Additionally, through analysis of peripheral blood from 49 Japanese patients with ESCC, Tamura et al. [20] found that the VEGF-634C/G genotype [an SNP in the $5^{\prime}$ untranslated region (UTR)] is predictive of better OS after treatment with definitive CRT. It has also been reported that genetic polymorphisms in the promoter region, 5'UTR and 3'UTR of the VEGF gene, are associated with VEGF levels in plasma and tumors [21], which suggests the VEGF-634C/G genotype may be related to the blood VEGF level.

XPA and XPC are important molecules involved in mediating nucleotide excision for DNA repair [22]. Yang et al. [23] used polymerase chain reaction (PCR) to analyze the genomic DNA isolated from peripheral leukocytes collected from 501 ESCC patients and demonstrated that patients with the XPA 5'UTR A/G and XPC K939Q C/C genotypes are at higher risk of mortality after treatment than patients with wild-type homozygous genotypes, and this effect was more pronounced in patients treated with neoadjuvant CRT followed by esophagectomy. 
Sato et al.: Novel Candidate Biomarkers of Chemoradiosensitivity in Esophagea

Squamous Cell Carcinoma: A Systematic Review

Table 2. Novel candidate biomarkers in tissue that are predictive of chemoradiosensitivity in ESCC

\begin{tabular}{|c|c|c|c|c|c|c|}
\hline Biomarker & Major function & $\begin{array}{l}\text { Sample } \\
\text { size }\end{array}$ & Method & $\begin{array}{l}\text { Predictive value of the } \\
\text { sensitivity }\end{array}$ & First author [Ref.] & Year \\
\hline \multicolumn{7}{|l|}{ Positive } \\
\hline ZNF695 & DNA repair & 104 & PCR & ZNF695 methylation (+) & Takahashi [28] & 2015 \\
\hline LOC285194 & long noncoding RNA & 55 & PCR & LOC285194 (+) & Tong [31] & 2014 \\
\hline REG1A & cell proliferation & 105 & IHC & REG1A (+) & Sato [35] & 2013 \\
\hline \multicolumn{7}{|l|}{ Negative } \\
\hline MDM2 & oncogene & 79 & $\mathrm{IHC}$ & MDM2 (-) & Okamoto [38] & 2015 \\
\hline \multirow[t]{2}{*}{ Nrf2 } & intracellular antioxidants & 46 & IHC & $\operatorname{Nrf}(-)$ & Kawasaki [41] & 2014 \\
\hline & & 33 & PCR & Nrf mutation (-) & Shibata [42] & 2011 \\
\hline Rad51 & DNA repair & 39 & IHC & Rad51 (-) & Nakanoko [44] & 2014 \\
\hline HIF- $1 \alpha$ & $\begin{array}{l}\text { homeostatic response to } \\
\text { hypoxia }\end{array}$ & 942 & meta-analysis & HIF-1 $\alpha(-)$ & Ping [46] & 2014 \\
\hline PC4 & DNA repair & 98 & IHC & PC4 (-) & Qian [48] & 2014 \\
\hline ERCC1 & DNA repair & 44 & IHC & ERCC1 (-) & Ge [50] & 2014 \\
\hline \multirow[t]{2}{*}{ EGFR } & cell proliferation & 47 & IHC & EGFR (-) & Gao [54] & 2014 \\
\hline & & 148 & PCR & $\begin{array}{l}\text { EGFR homozygous long } \\
\text { allele (L/L) }\end{array}$ & Lee [55] & 2011 \\
\hline PITX2 & embryonic development & 424 & IHC & PITX2 (-) & Zhang [58] & 2013 \\
\hline PinX1 & telomere stability & 157 & IHC & PinX1 (-) & Qian [60] & 2013 \\
\hline BRCA1 & DNA repair & 144 & PCR & BRCA1 (-) & Gao [64] & 2013 \\
\hline p53 & tumor suppressor & 1,497 & meta-analysis & p53 low or wild type & Zhang [65] & 2013 \\
\hline mTOR & cell proliferation & 77 & IHC & mTOR $(-)$ & Li [67] & 2012 \\
\hline $\mathrm{COX}-2$ & PG synthesis & 58 & $\mathrm{IHC}$ & $\operatorname{COX} 2(-)$ & Akutsu [72] & 2011 \\
\hline $\begin{array}{l}\text { Combination } \\
\text { MMP1, LIMCH1, } \\
\text { C1orf226 }\end{array}$ & ECM degradation & 60 & PCR & $\begin{array}{l}\text { MMP1 (+), LIMCH1 (-), } \\
\text { C1orf226 (-) }\end{array}$ & Wen [75] & 2014 \\
\hline $\begin{array}{l}\text { Ki-67, NF-кB, } \\
\text { HER2, ER }\end{array}$ & cell proliferation & 10 & IHC & $\begin{array}{l}\text { Ki-67 (+), NF-кB (-), } \\
\text { HER2 (-), ER (-) }\end{array}$ & Shibata [78] & 2013 \\
\hline $\begin{array}{l}\text { MLH1,FANCD2, } \\
\text { PARP1, XPF, PAR }\end{array}$ & DNA repair & 79 & IHC & MLH1 (+), FANCD2 (-) & Alexander [82] & 2012 \\
\hline PTCH1, GLI-1 & development of organs & 100 & IHC & PTCH (-), GLI-1 (-) & Zhu [84] & 2011 \\
\hline
\end{tabular}

$\mathrm{IHC}=$ Immunohistochemistry; $\mathrm{ECM}$ = extracellular matrix; PG = prostaglandin .

Interleukin-6 receptor (IL-6R; also known as CD126) is a cell membrane cognate receptor for the inflammatory cytokine IL-6. Soluble (s)IL-6R is an alternatively spliced or proteolytically cleaved form of IL-6R that lacks the transmembrane domain. Secreted sIL-6R binds to IL-6, and the resultant sIL-6R/IL-6 complex evokes intracellular signaling [24]. Makuuchi et al. [25] showed that the serum sIL-6R levels were significantly higher in 30 patients who failed to achieve a pathological complete response (pCR), and multivariate analysis indicated that a high sIL-6R level is one of several significant independent predictors of an unfavorable outcome after preoperative CRT followed by esophagectomy.

\section{Combination of Biomarkers in Blood Examination}

Metabolomics is a powerful approach that enables quantitative measurement of global changes in the metabolic profiles of individuals in response to disease or treatment through noninvasive analysis of biofluids [26]. Using metabolomics, Xu et al. [27] observed that the 
levels of three metabolites, lysoPC(16:1), octanoylcarnitine and decanoylcarnitine, correlated closely with treatment and showed a tendency toward gradual recovery to healthy levels in the responder group. In the nonresponder group, no obvious changes in the levels of these metabolites were observed over the treatment course. It was therefore concluded that these biomarkers may be useful in diagnosis, as well as for monitoring therapeutic responses and predicting outcomes in ESCC.

\section{Positive Biomarkers in Tissue Examination}

DNA methylation status reportedly correlates with the clinicopathological features of many types of cancer. Using genome-wide methylation analysis with 7 responders and 8 nonresponders, Takahashi et al. [28] showed that ZNF695 methylation in ESCC tissue samples is an independent factor predictive of the response to definitive CRT. Because ZNF695 is a zinc finger protein and a likely transcription factor, they speculated that ZNF695 is predictive of outcome because it regulates the expression of genes involved in the repair of DNA damage introduced by CRT. Recently, it was reported that ZNF695 splice variants were highly expressed in ovarian cancer [29]. Although it is still unknown whether these splice variants are associated with aggressive behavior in ovarian cancer, it is possible that through loss of ZNF695 function, cancer cells become highly proliferative, resulting in resistance to therapy. There are no other reports of a relationship between ZNF695 and cancer.

Long noncoding RNAs (lncRNAs) represent a new class of non-protein-coding RNAs, which are longer than 200 bases and do not function as templates for protein synthesis. Studies have also shown that lncRNAs play a critical role in the development and progression of cancers, including ESCC [30]. Tong et al. [31] reported that low LOC285194 expression correlated significantly with a poorer CRT response and that patients with low LOC285194 expression had significantly shorter DFS and OS. Moreover, multivariable analysis identified low LOC285194 expression as an independent prognostic factor for CRT response, DFS and OS. Though numerous reports on the relationship between lncRNAs and esophageal cancer have been published, there are no IncRNA candidates other than LOC285194 whose expression relates to chemoradiosensitivity.

REG1A was originally isolated from pancreatic islet $\beta$-cells as an endogenous growth factor [32]. We previously reported that REG1A is a reliable sensitivity marker in ESCC patients (clinical T4) treated with neoadjuvant CRT [33] or definitive CRT [34]. We also recently showed that among stage II and III ESCC patients treated with esophagectomy followed by adjuvant chemotherapy, OS, DSS and DFS were all significantly better in REG1A-positive than REG1Anegative patients [35]. Additionally, we demonstrated that REG1A gene transfection enhances radiosensitivity by affecting the MAPK pathway in ESCC cells [36]. New studies aimed at determining whether REG1A also works as a reliable sensitivity marker in stage II and III ESCC patients treated with neoadjuvant CRT followed by esophagectomy are now ongoing. However, the number of patients in our institution is limited, so accumulation of strong evidence will take time. Studies of REG1A from other institutions treating different populations will facilitate clinical application of REG1A as a reliable sensitivity marker in ESCC.

\section{Negative Biomarkers in Tissue Examination}

MDM2 promotes tumor formation by targeting tumor suppressor genes (e.g., p53 and p16), and its overexpression has been detected in a variety of different cancers [37]. Okamoto et al. [38] reported that MDM2 positivity in pretreatment biopsy specimens was significantly 
Sato et al.: Novel Candidate Biomarkers of Chemoradiosensitivity in Esophageal

Squamous Cell Carcinoma: A Systematic Review

higher in the failure group than in the CR group among cStageIII ESCC patients treated with definitive CRT. In addition, it was reported that the MDM2 inhibitor Nutlin-3 increased the radiosensitivity of laryngeal squamous cell carcinoma [39]. Therefore, it may be clinically worthwhile to attempt a clinical study of this inhibitor in patients with ESCC.

Nrf2 was originally identified as a critical regulator of intracellular antioxidants, and recent studies have shown that strong Nrf2 expression gives cancer cells a survival advantage during anticancer chemotherapy and radiotherapy [40]. Kawasaki et al. [41] found a significant correlation between Nrf2 expression in biopsy specimens before CRT and an unfavorable response to neoadjuvant CRT. In addition, Shibata et al. [42] showed that Nrf2 gene mutation was associated with tumor recurrence and a poor prognosis, and that downregulation of the mutant Nrf2 increases the sensitivity of ESCC cells to 5-fluorouracil and radiation. Strong Nrf2 expression and its gene mutation thus appear to indicate resistance to CRT.

Rad51 plays an important role in the repair of DNA double-strand breaks through homologous recombination, thereby decreasing sensitivity to chemotherapy and radiotherapy [43]. Nakanoko et al. [44] reported that a pCR was more frequently observed in Rad51-negative than Rad51-positive ESCC patients treated with neoadjuvantCRT followed by esophagectomy. Rad51 also interacts with a variety of tumor suppressor proteins, including p53, BRCA1 and BRCA2, indicating a possible association of Rad51 with the process of tumorigenesis and resistance to CRT. However, the role of Rad51 in these processes is not yet understood.

HIF- $1 \alpha$ is a key transcription factor that functions in response to hypoxic stress by regulating genes involved in maintaining oxygen homeostasis [45]. Recently published studies on the prognostic value of HIF-1 $\alpha$ overexpression in ESCC are conflicting and heterogeneous. However, Ping et al. [46] performed a meta-analysis that included 12 studies with a total of 942 ESCC patients and found that HIF-1 $\alpha$ overexpression was a potent predictor of a poor prognosis and resistance to CRT in ESCC. At present, HIF-1 $\alpha$ appears to be one of the most promising candidate markers predictive of the response to CRT in ESCC.

Human positive cofactor 4 (PC4) plays important roles in gene transcription and replication, DNA damage repair, chromatin organization and cell cycle progression [47]. Qian et al. [48] showed that high expression of PC4 positively correlates with resistance to CRT and is an independent predictor of short DSS in ESCC patients who receive definitive CRT with cisplatin (CDDP)-based chemotherapy. However, there are no other reports on the relationship between PC4 and ESCC at this time.

ERCC1 appears to be involved in repairing DNA damage induced by chemotherapy and radiotherapy in cancer tissue [49]. Ge et al. [50] reported that low ERCC1 expression was an independent predictor of better OS in patients with locally advanced ESCC treated with neoadjuvant CRT followed by esophagectomy, and those findings have been confirmed by others $[51,52]$.

Diffuse epidermal growth factor receptor (EGFR) expression is reportedly associated with reduced OS in patients with locally advanced ESCC [53]. In addition, Gao et al. [54] found a significant correlation between EGFR overexpression and poor OS among patients with locally advanced ESCC receiving definitive CRT, while Lee et al. [55] observed that the EGFR genotype is predictive of clinical outcome after neoadjuvant CRT followed by esophagectomy. When the accumulating evidence becomes sufficient, EGFR-TKI may also be considered for clinical application as a treatment option for ESCC as well as non-small cell lung cancer.

It is well known that PITX2 is crucial for normal embryonic development [56]. In recent studies, PITX2 was identified as a target gene in the Wnt/ $\beta$-catenin and hedgehog/TGF pathways, which regulate several oncogenes, including MYC, cyclin D1 and cyclin D2 [57]. Zhang et al. [58] found that strong PITX2 expression was more frequently observed in CRTresistant patients than in those responsive to CRT, and that strong PITX2 expression was associated with poor DSS among ESCC patients treated with definitive CRT. They also demon- 
Sato et al.: Novel Candidate Biomarkers of Chemoradiosensitivity in Esophageal

Squamous Cell Carcinoma: A Systematic Review

strated that PITX2 knockdown substantially increased the sensitivity of ESCC cells to radiation or CDDP in vitro. However, there are currently no other reports on the relationship between PITX2 and responsiveness to CRT or prognosis.

The telomerase-/telomere-interacting protein PinX1 contributes to telomere maintenance and tumorigenicity, and several studies have documented that telomerase activity is a determinant of cytotoxic drug sensitivity in ESCC [59]. Qian et al. [60] showed that strong expression of PinX1 correlated positively with ESCC resistance to CRT and was a strong and independent predictor of short DSS in ESCC patients. Moreover, PinX1 knockdown substantially increased the therapeutic efficacy of radiation against ESCC cells in vitro and in vivo, and ectopic overexpression of PinX1 dramatically enhanced ESCC cell resistance to radiotherapy. On the other hand, Zuo et al. [61] reported that PinX1 had the opposite effect on carcinogenesis among esophageal epithelial cells.

BRCA1 was first identified as one of the genes that conferred genetic predisposition to early-onset breast and ovarian cancer [62]. However, increasing numbers of reports have shown that BRCA1 is also a regulator of chemotherapy-induced DNA damage in cancer cells [63]. Gao et al. [64] reported that low BRCA1 transcription correlated with a higher response rate and longer OS in ESCC patients receiving CDDP-based chemotherapy or CRT. On the other hand, low BRCA1 transcription also correlated with shorter OS in patients receiving docetaxelbased chemotherapy or CRT. BRCA1 may thus be an indicator that enables us to appropriately select CDDP-based or docetaxel-based therapy for more individualized treatment.

Though p53 is reportedly useful for predicting the response to CRT in ESCC patients, it remains controversial. In 2013, Zhang et al. [65] reported a meta-analysis that included 28 studies involving 1,497 ESCC patients. They reported that low expression of the p53 protein and/or the wild-type p53 gene was associated with a good response to both chemotherapy and CRT in ESCC. Additionally, patients carrying the wild-type form of p53 had a high rate of pCR to neoadjuvant CRT. These results provide further evidence that p53 would be a reliable biomarker of a positive response to CRT in ESCC.

Clinicians are beginning to use mTOR inhibitors (e.g., temsirolimus and everolimus) in the treatment of cancer as well as several age-associated diseases, including neurodegenerative diseases such as Alzheimer's disease and Parkinson's disease [66]. Li et al. [67] showed that levels of phosphorylated mTOR are independently associated with the response to CRT and prognosis in patients treated with neoadjuvant CRT followed by esophagectomy. They also demonstrated that mTOR inhibition sensitizes ESCC cells to chemotherapy in vitro. Two other reports have confirmed the connection between mTOR inhibition and improved chemosensitivity in ESCC $[68,69]$. Notably, the mTOR pathway leads to upregulation of HIF-1 $\alpha$, the effects of which have been discussed above [70].

COX1 and COX2 play crucial roles in prostaglandin synthesis. COX1 is expressed in normal tissues to maintain homeostatic function. COX2, by contrast, is primarily expressed in cancers, including ESCC [71], and Akutsu et al. [72] found that COX2 expression is predictive of resistance to neoadjuvant CRT in ESCC patients. In addition, Okamura et al. [73] showed that ESCC cells transfected with COX2 were resistant to CDDP + 5-fluorouracil treatment.

\section{Combination of Biomarkers in Tissue Examination}

MMP1 is a member of the matrix metalloproteinase (MMP) family and is reportedly involved in the response to cancer drug therapy through its regulation of vascular leakage [74]. Wen et al. [75] analyzed gene expression in pretreatment biopsy specimens from 28 ESCC patients who received neoadjuvant CRT and esophagectomy. The gene profiling identified 10 differentially expressed genes with more than a twofold difference between patients 
with a pCR and those with less than a pCR. Using a combination of 3 genes, MMP1, LIMCH1 and C1orf226, ESCC patients could be effectively and reproducibly classified with respect to their responsiveness to neoadjuvant CRT.

Ki-67 (MIB-1) monoclonal antibody recognizes proliferating cells in the G1, S, G2 and M phases of the cell cycle [76]. The correlation between a high MIB-1 index and poor prognosis is well known in breast cancer [77]. By contrast, Shibata et al. [78] reported that ESCC patients with a higher MIB-1 index showed significantly better OS following definitive CRT than patients with a lower index. It was also reported from a study of 56 ESCC patients that overexpression of MIB-1 is a significant factor for a complete endoscopic response following definitive CRT [79]. The correlation between MIB-1 expression and a good response to CRT may be explained by the fact that MIB-1 expression is a marker of cellular proliferation.

$N F-\kappa B$ is a protein complex that controls DNA transcription. Blocking NF- $\kappa B$ appears to cause tumor cells to stop proliferating or become more sensitive to antitumor agents [80]. HER2 is a member of the EGFR family that is involved in the complex regulation of cell growth, proliferation and survival. In addition to breast cancer, HER2 overexpression and gene amplification have also been reported in several other cancers, including ESCC [81]. Estrogen promotes tumor cell proliferation by acting via the estrogen receptor, and that proliferation appears to be enhanced in tumor cells overexpressing HER2. Shibata et al. [78] also showed that low levels of NF- $\mathrm{KB}, \mathrm{HER} 2$ and estrogen receptor were good prognostic factors following definitive CCRT.

Alexander et al. [82] analyzed other candidate biomarkers selected from multiple DNA repair pathways. They showed that the expression patterns of DNA repair proteins, including XPF, FANCD2, PAR, MLH1 and PARP1, are associated with the pathological response to neoadjuvant CRT and OS. ESCC patients with high MLH1 and low FANCD2 expression tended to have better OS, but the effect did not reach statistical significance due to the small number of patients.

The hedgehog signaling pathway plays a critical role in the development of various organs, and aberrant activation of this pathway was recently observed in several cancers, including ESCC [83]. Zhu et al. [84] showed that the expression status of two hedgehog components, PTCH1 and the glioma-associated oncogene GLI-1, were independent prognostic factors associated with poor DSS and OS.

\section{Problems of Novel Candidate Biomarkers}

In this systematic review, we included 32 recently reported studies and enumerated 41 candidate biomarkers that are potentially predictive of chemoradiosensitivity in ESCC. We found meta-analyses that analyzed 12 studies of HIF- $1 \alpha$ that included a total of 942 patients [46] and 28 studies of p53 that included a total of 1,497 patients [65]. At present, HIF-1 $\alpha$ and p53 appear to be the most promising candidate biomarkers predictive of chemoradiosensitivity in ESCC. However, for even these candidate biomarkers to be useful in clinical application, additional strong evidence of their efficacy will be required. For example, HIF-1 $\alpha$ overexpression was significantly associated with poor OS and DFS, and with a poor response to CRT in a meta-analysis [46]. However, HIF-1 $\alpha$ overexpression was also significantly associated with stage, lymph node metastasis, depth of invasion, lymphatic invasion and distant metastasis, and it is to be expected that more advanced ESCCs are related to a poor outcome and poor response to CRT. Therefore, subgroup analyses among different $\mathrm{T}$ statuses, $\mathrm{N}$ statuses, $\mathrm{M}$ statuses and stages, as well as additional results from large prospective studies performed with different populations will be needed to confirm the reliability of HIF-1 $\alpha$ as an independent sensitivity marker suitable for clinical application. 
There are 9 articles on candidate biomarkers in blood, samples of which are easily collected and analyzed using blood PCR, serum PCR and rapid resolution liquid chromatography-mass spectrometry. Blood-borne biomarkers appear to reflect global changes in the body containing ESCC tissue. It is thus widely expected that additional evidence for candidate biomarkers in blood will be produced in the future. On the other hand, there are problems that must be overcome before pretreatment biopsy tissue, which is commonly used today, can be used to predict CRT outcomes. This is because biopsy samples show only a small fraction of an ESCC tumor, and they are quite heterogeneous.

\section{Conclusion}

Some of the candidate biomarkers named in this review have been studied at different institutions in different populations, but are still a long way from clinical application. Although it is important to continue to search for novel candidates, it will also be important to continue to obtain strong evidence to determine the suitability for clinical application of candidates already identified.

\section{Acknowledgement}

This work was supported by the Takeda Science Foundation.

\section{Disclosure Statement}

The authors have no conflicts of interest to declare.

\section{References}

1 Montgomery EA: Oesophageal Cancer; in Stewart BW, Wild CP (eds): World Cancer Report 2014. Geneva, World Health Organization, pp 528-543.

2 Ando N, Ozawa S, Kitagawa Y, et al: Improvement in the results of surgical treatment of advanced squamous esophageal carcinoma during 15 consecutive years. Ann Surg 2002;232:225-232.

3 Law S, Kwong DL, Kwok KF, et al: Improvement in treatment results and long-term survival of patients with esophageal cancer: impact of chemoradiation and change in treatment strategy. Ann Surg 2003;238:339-347.

4 Ando N, Iizuka T, Ide H, et al: Surgery plus chemotherapy compared with surgery alone for localized squamous cell carcinoma of the thoracic esophagus: a Japan Clinical Oncology Group Study - JCOG9204. J Clin Oncol 2003;21:4592-4596.

5 Kato K, Muro K, Minashi K, et al: Phase II study of chemoradiotherapy with 5-fluorouracil and cisplatin for stage II-III esophageal squamous cell carcinoma: JCOG trial (JCOG 9906). Int J Radiat Oncol Biol Phys 2011;81: 684-690.

6 Ando $\mathrm{N}$, Kato $\mathrm{H}$, Igaki $\mathrm{H}$, et al: A randomized trial comparing postoperative adjuvant chemotherapy with cisplatin and 5-fluorouracil versus preoperative chemotherapy for localized advanced squamous cell carcinoma of the thoracic esophagus (JCOG9907). Ann Surg Oncol 2012;19:68-74.

7 Sanuki N, Ishikura S, Shinoda M, et al: Radiotherapy quality assurance review for a multi-center randomized trial of locally advanced esophageal cancer: the Japan Clinical Oncology Group (JCOG) trial 0303. Int J Clin Oncol 2012;17:105-111.

8 Sato Y, Motoyama S, Nanjo H, et al: REG1A expression status suggests chemosensitivity among advanced thoracic esophageal squamous cell carcinoma patients treated with esophagectomy followed by adjuvant chemotherapy. Ann Surg Oncol 2013;20:3044-3051.

9 Okumura H, Uchikado Y, Setoyama T, et al: Biomarkers for predicting the response of esophageal squamous cell carcinoma to neoadjuvant chemoradiation therapy. Surg Today 2014;44:421-428.

10 Kuwano H, Nishimura Y, Oyama T, et al: Guidelines for Diagnosis and Treatment of Carcinoma of the Esophagus April 2012 edited by the Japan Esophageal Society. Esophagus 2015;12:1-30. 
11 Marzec KA, Baxter RC, Martin JL: Targeting insulin-like growth factor binding protein-3 signaling in triplenegative breast cancer. Biomed Res Int 2015;2015:638526.

12 Zhao L, Chi F, Xi M, et al: Polymorphisms of insulin-like growth factor binding protein-3 as a predictor for risk and patient survival in esophageal squamous cell carcinoma. Biomed Pharmacother 2015;74:148-152.

13 Yoshino K, Motoyama S, Koyota S, et al: IGFBP3 and BAG1 enhance radiation-induced apoptosis in squamous esophageal cancer cells. Biochem Biophys Res Commun 2011;404:1070-1075.

14 Aggarwal BB: Signaling pathways of the TNF superfamily: a double-edged sword. Nat Rev Immunol 2003;3: 745-756.

15 Omatsu H, Kuwahara A, Yamamori M, et al: TNF- $\alpha-857 \mathrm{C}>\mathrm{T}$ genotype is predictive of clinical response after treatment with definitive 5-fluorouracil/cisplatin-based chemoradiotherapy in Japanese patients with esophageal squamous cell carcinoma. Int J Med Sci 2013;10:1755-1760.

16 Hamai Y, Hihara J, Taomoto J, et al: Hemoglobin level influences tumor response and survival after neoadjuvant chemoradiotherapy for esophageal squamous cell carcinoma. World J Surg 2014;38:2046-2051.

17 Hamai Y, Hihara J, Emi M, et al: Treatment outcomes and prognostic factors for thoracic esophageal cancer with clinical evidence of adjacent organ invasion. Anticancer Res 2013;33:3495-3502.

18 Kleespies A, Guba M, Jauch KW, et al: Vascular endothelial growth factor in esophageal cancer. J Surg Oncol 2004;87:95-104.

19 Cheng JC, Graber MS, Hsu FM, et al: High serum levels of vascular endothelial growth factor-A and transforming growth factor- $\beta 1$ before neoadjuvant chemoradiotherapy predict poor outcomes in patients with esophageal squamous cell carcinoma receiving combined modality therapy. Ann Surg Oncol 2014;21:23612368.

20 Tamura T, Kuwahara A, Yamamori M, et al: VEGF -634C/G genotype is predictive of long-term survival after treatment with a definitive 5-fluorouracil/cisplatin-based chemoradiotherapy in Japanese patients with esophageal squamous cell carcinoma. Int J Med Sci 2012;9:833-837.

21 Schneider BP, Radovich M, Miller KD: The role of vascular endothelial growth factor genetic variability in cancer. Clin Cancer Res 2009;15:5297-5302.

22 Li CL, Golebiowski FM, Onishi Y, et al: Tripartite DNA lesion recognition and verification by XPC, TFIIH, and XPA in nucleotide excision repair. Mol Cell 2015;59:1025-1034.

23 Yang PW, Hsieh CY, Kuo FT, et al: The survival impact of XPA and XPC genetic polymorphisms on patients with esophageal squamous cell carcinoma. Ann Surg Oncol 2013;20:562-571.

24 Jones SA, Scheller J, Rose-John S: Therapeutic strategies for the clinical blockade of IL-6/gp130 signaling. J Clin Invest 2011;121:3375-3383.

25 Makuuchi Y, Honda K, Osaka Y, et al: Soluble interleukin-6 receptor is a serum biomarker for the response of esophageal carcinoma to neoadjuvant chemoradiotherapy. Cancer Sci 2013;104:1045-1051.

26 Ganti S, Taylor SL, Abu Aboud O, et al: Kidney tumor biomarkers revealed by simultaneous multiple matrix metabolomics analysis. Cancer Res 2012;72:3471-3479.

$27 \mathrm{Xu} \mathrm{J}$, Chen Y, Zhang R, et al: Global and targeted metabolomics of esophageal squamous cell carcinoma discovers potential diagnostic and therapeutic biomarkers. Mol Cell Proteomics 2013;12:1306-1318.

28 Takahashi T, Yamahsita S, Matsuda Y, et al: ZNF695 methylation predicts a response of esophageal squamous cell carcinoma to definitive chemoradiotherapy. J Cancer Res Clin Oncol 2015;141:453-463.

29 Juárez-Méndez S, Zentella-Dehesa A, Villegas-Ruíz V, et al: Splice variants of zinc finger protein 695 mRNA associated to ovarian cancer. J Ovarian Res 2013;6:61.

30 Chen FJ, Sun M, Li SQ, et al: Upregulation of the long non-coding RNA HOTAIR promotes esophageal squamous cell carcinoma metastasis and poor prognosis. Mol Carcinog 2013;52:908-915.

31 Tong YS, Zhou XL, Wang XW, et al: Association of decreased expression of long non-coding RNA LOC285194 with chemoradiotherapy resistance and poor prognosis in esophageal squamous cell carcinoma. J Transl Med 2014;12:233.

32 Terazono K, Yamamoto H, Takasawa S, et al: A novel gene activated in regenerating islets. J Biol Chem 1988; 263:2111-2114.

33 Motoyama S, Sugiyama T, Ueno Y, et al: REG I expression predicts long-term survival among locally advanced thoracic squamous cell esophageal cancer patients treated with neoadjuvant chemoradiotherapy followed by esophagectomy. Ann Surg Oncol 2006;13:1724-1731.

34 Hayashi K, Motoyama S, Sugiyama T, et al: REG Ialpha is a reliable marker of chemoradiosensitivity in squamous cell esophageal cancer patients. Ann Surg Oncol 2008;15:1224-1231.

35 Sato Y, Motoyama S, Nanjo H, et al: REG1A expression status suggests chemosensitivity among advanced thoracic esophageal squamous cell carcinoma patients treated with esophagectomy followed by adjuvant chemotherapy. Ann Surg Oncol 2013;20:3044-3051.

36 Wakita A, Motoyama S, Sato Y, et al: REG I $\alpha$ activates c-Jun through MAPK pathways to enhance the radiosensitivity of squamous esophageal cancer cells. Tumour Biol 2015;36:5249-5254.

37 Park HS, Park JM, Park S, et al: Subcellular localization of Mdm2 expression and prognosis of breast cancer. Int J Clin Oncol 2014;19:842-851.

38 Okamoto H, Fujishima F, Kamei T, et al: Murine double minute 2 predicts response of advanced esophageal squamous cell carcinoma to definitive chemoradiotherapy. BMC Cancer 2015;15:208.

39 Arya AK, El-Fert A, Devling T, et al: Nutlin-3, the small-molecule inhibitor of MDM2, promotes senescence and radiosensitises laryngeal carcinoma cells harbouring wild-type p53. Br J Cancer 2010;103:186-195. 
40 Cho JM, Manandhar S, Lee HR, et al: Role of the Nrf2-antioxidant system in cytotoxicity mediated by anticancer cisplatin: implication to cancer cell resistance. Cancer Lett 2008;260:96-108.

41 Kawasaki Y, Okumura H, Uchikado Y, et al: Nrf2 is useful for predicting the effect of chemoradiation therapy on esophageal squamous cell carcinoma. Ann Surg Oncol 2014;21:2347-2352.

42 Shibata T, Kokubu A, Saito S, et al: NRF2 mutation confers malignant potential and resistance to chemoradiation therapy in advanced esophageal squamous cancer. Neoplasia 2011;13:864-873.

43 Henning W, Stürzbecher HW: Homologous recombination and cell cycle checkpoints: Rad51 in tumour progression and therapy resistance. Toxicology 2003;193:91-109.

44 Nakanoko T, Saeki H, Morita M, et al: Rad51 expression is a useful predictive factor for the efficacy of neoadjuvant chemoradiotherapy in squamous cell carcinoma of the esophagus. Ann Surg Oncol 2014;21:597-604.

45 Zhan L, Huang C, Meng XM, et al: Hypoxia-inducible factor-1alpha in hepatic fibrosis: a promising therapeutic target. Biochimie 2015;108:1-7.

46 Ping W, Sun W, Zu Y, et al: Clinicopathological and prognostic significance of hypoxia-inducible factor-1 $\alpha$ in esophageal squamous cell carcinoma: a meta-analysis. Tumour Biol 2014;35:4401-4409.

47 Das C, Hizume K, Batta K, et al: Transcriptional coactivator PC4, a chromatin-associated protein, induces chromatin condensation. Mol Cell Biol 2006;26:8303-8315.

48 Qian D, Zhang B, Zeng XL, et al: Inhibition of human positive cofactor 4 radiosensitizes human esophageal squmaous cell carcinoma cells by suppressing XLF-mediated nonhomologous end joining. Cell Death Dis 2014; 5:e1461.

49 Sakano S, Ogawa S, Yamamoto Y, et al: ERCC1 and XRCC1 expression predicts survival in bladder cancer patients receiving combined trimodality therapy. Mol Clin Oncol 2013;1:403-410.

$50 \mathrm{Ge} \mathrm{H}, \mathrm{Lu}$ Y, Chen Y, et al: ERCC1 expression and tumor regression predict survival in esophageal squamous cell carcinoma patients receiving combined trimodality therapy. Pathol Res Pract 2014;210:656-661.

51 Fareed KR, Al-Attar A, Soomro IN, et al: Tumour regression and ERCC1 nuclear protein expression predict clinical outcome in patients with gastro-oesophageal cancer treated with neoadjuvant chemotherapy. $\mathrm{Br} \mathrm{J}$ Cancer 2010;102:1600-1607.

52 Kim MK, Cho KJ, Kwon GY, et al: ERCC1 predicting chemoradiation resistance and poor outcome in oesophageal cancer. Eur J Cancer 2008;44:54-60.

53 Gibault L, Metges JP, Conan-Charlet V, et al: Diffuse EGFR staining is associated with reduced overall survival in locally advanced oesophageal squamous cell cancer. Br J Cancer 2005; 93:107-115.

54 Gao Z, Meng X, Mu D, et al: Prognostic significance of epidermal growth factor receptor in locally advanced esophageal squamous cell carcinoma for patients receiving chemoradiotherapy. Oncol Lett 2014;7:11181122.

55 Lee JM, Yang SY, Yang PW, et al: Polymorphism in epidermal growth factor receptor intron 1 predicts prognosis of patients with esophageal cancer after chemoradiation and surgery. Ann Surg Oncol 2011;18:20662073.

56 Bamforth SD, Bragança J, Farthing CR, et al: Cited2 controls left-right patterning and heart development through a Nodal-Pitx2c pathway. Nat Genet 2004;36:1189-1196.

57 Huang Y, Guigon CJ, Fan J, et al: Pituitary homeobox 2 (PITX2) promotes thyroid carcinogenesis by activation of cyclin D2. Cell Cycle 2010;9:1333-1341.

58 Zhang JX, Tong ZT, Yang L, et al: PITX2: a promising predictive biomarker of patients' prognosis and chemoradioresistance in esophageal squamous cell carcinoma. Int J Cancer 2013;132:2567-2577.

59 Zhang X, Komaki R, Wang L, et al: Treatment of radioresistant stem-like esophageal cancer cells by an apoptotic gene-armed, telomerase-specific oncolytic adenovirus. Clin Cancer Res 2008;14:2813-2823.

60 Qian D, Zhang B, He LR, et al: The telomere/telomerase binding factor PinX1 is a new target to improve the radiotherapy effect of oesophageal squamous cell carcinomas. J Pathol 2013;229:765-774.

61 Zuo J, Wang DH, Zhang YJ, et al: Expression and mechanism of PinX1 and telomerase activity in the carcinogenesis of esophageal epithelial cells. Oncol Rep 2013;30:1823-1831.

62 Kennedy RD, Quinn JE, Johnston PG, etal: BRCA1: mechanisms of inactivation and implications for management of patients. Lancet 2002;360:1007-1014.

63 Tassone P, Tagliaferri P, Perricelli A, et al: BRCA1 expression modulates chemosensitivity of BRCA1-defective HCC1937 human breast cancer cells. Br J Cancer 2003;88:1285-1291.

64 Gao Y, Zhu J, Zhang X, Wu Q, et al: BRCA1 mRNA expression as a predictive and prognostic marker in advanced esophageal squamous cell carcinoma treated with cisplatin- or docetaxel-based chemotherapy/chemoradiotherapy. PLoS One 2013;8:e52589.

65 Zhang SS, Huang QY, Yang H, et al: Correlation of p53 status with the response to chemotherapy-based treatment in esophageal cancer: a meta-analysis. Ann Surg Oncol 2013;20:2419-2427.

66 Wang C, Yu JT, Miao D, et al: Targeting the mTOR signaling network for Alzheimer's disease therapy. Mol Neurobiol 2014;49:120-135.

67 Li SH, Huang EY, Lu HI, et al: Phosphorylated mammalian target of rapamycin expression is associated with the response to chemoradiotherapy in patients with esophageal squamous cell carcinoma. J Thorac Cardiovasc Surg 2012;144:1352-1359.

68 Hou G, Yang S, Zhou Y, et al: Targeted inhibition of mTOR signaling improves sensitivity of esophageal squamous cell carcinoma cells to cisplatin. J Immunol Res 2014;2014:845763. 
69 Huang Y, Xi Q, Chen Y, et al: A dual mTORC1 and mTORC2 inhibitor shows antitumor activity in esophageal squamous cell carcinoma cells and sensitizes them to cisplatin. Anticancer Drugs 2013;24:889-898.

70 Nishikawa T, Takaoka M, Ohara T, et al: Antiproliferative effect of a novel mTOR inhibitor temsirolimus contributes to the prolonged survival of orthotopic esophageal cancer-bearing mice. Cancer Biol Ther 2013; 14:230-236.

71 Shamma A, Yamamoto H, Doki Y, et al: Up-regulation of cyclooxygenase-2 in squamous carcinogenesis of the esophagus. Clin Cancer Res 2000;6:1229-1238.

72 Akutsu Y, Hanari N, Yusup G, et al: COX2 expression predicts resistance to chemoradiotherapy in esophageal squamous cell carcinoma. Ann Surg Oncol 2011;18:2946-2951.

73 Okamura H, Fujiwara H, Umehara S, et al: COX-2 overexpression induced by gene transfer reduces sensitivity of TE13 esophageal carcinoma cells to 5-fluorouracil and cisplatin. Anticancer Res 2013;33:537-542.

74 Nakasone ES, Askautrud HA, Kees T, et al: Imaging tumor-stroma interactions during chemotherapy reveals contributions of the microenvironment to resistance. Cancer Cell 2012;21:488-503.

75 Wen J, Yang H, Liu MZ, et al: Gene expression analysis of pretreatment biopsies predicts the pathological response of esophageal squamous cell carcinomas to neo-chemoradiotherapy. Ann Oncol 2014;25:17691774.

76 Scholzen T, Gerdes J: The Ki-67 protein: from the known and the unknown. J Cell Physiol 2000;182:311-322.

77 Yerushalmi R, Woods R, Ravdin PM, et al: Ki67 in breast cancer: prognostic and predictive potential. Lancet Oncol 2010;11:174-183.

78 Shibata-Kobayashi S, Yamashita H, Okuma K, et al: Correlation among 16 biological factors [p53, p21(waf1), MIB-1 (Ki-67), p16(INK4A), cyclin D1, E-cadherin, Bcl-2, TNF- $\alpha$, NF- $\kappa$ B, TGF- $\beta$, MMP-7, COX-2, EGFR, HER2/ neu, ER, and HIF-1 $\alpha$ ] and clinical outcomes following curative chemoradiation therapy in 10 patients with esophageal squamous cell carcinoma. Oncol Lett 2013;5:903-910.

79 Ressiot E, Dahan L, Liprandi A, et al: Predictive factors of the response to chemoradiotherapy in esophageal cancer. Gastroenterol Clin Biol 2008;32:567-577.

80 Escárcega RO, Fuentes-Alexandro S, García-Carrasco M, et al: The transcription factor nuclear factor-kappa B and cancer. Clin Oncol (R Coll Radiol) 2007;19:154-161.

81 Akamatsu M, Matsumoto T, Oka K, et al: c-erbB-2 oncoprotein expression related to chemoradioresistance in esophageal squamous cell carcinoma. Int J Radiat Oncol Biol Phys 2003;57:1323-1327.

82 Alexander BM, Wang XZ, Niemierko A, et al: DNA repair biomarkers predict response to neoadjuvant chemoradiotherapy in esophageal cancer. Int J Radiat Oncol Biol Phys 2012;83:164-171.

83 Isohata N, Aoyagi K, Mabuchi T, et al: Hedgehog and epithelial-mesenchymal transition signaling in normal and malignant epithelial cells of the esophagus. Int J Cancer 2009;125:1212-1221.

84 Zhu W, You Z, Li T, et al: Correlation of hedgehog signal activation with chemoradiotherapy sensitivity and survival in esophageal squamous cell carcinomas. Jpn J Clin Oncol 2011;41:386-393. 